\title{
Focal Segmental Glomerulosclerosis: State-of-the-Art and Clinical Perspective
}

\author{
Amir Shabaka Ana Tato Ribera Gema Fernández-Juárez \\ Nephrology Department, Hospital Universitario Fundación Alcorcón, Madrid, Spain
}

\section{Keywords}

Focal segmental glomerulosclerosis · Diagnosis · Treatment

\begin{abstract}
Focal segmental glomerulosclerosis (FSGS) is a histological pattern of glomerular injury, rather than a single disease, that is caused by diverse clinicopathological entities with different mechanisms of injury with the podocyte as the principal target of lesion, leading to the characteristic sclerotic lesions in parts (i.e., focal) of some (i.e., segmental) glomeruli. The lesion of FSGS has shown an increasing prevalence over the past few decades and is considered the most common glomerular cause leading to ESKD. Primary FSGS, which usually presents with nephrotic syndrome, is thought to be caused by circulating permeability factors that have a main role in podocyte foot process effacement. Secondary forms of FSGS include maladaptive FSGS secondary to glomerular hyperfiltration such as in obesity or in cases of loss in nephron mass, virus-associated FSGS, and drugassociated FSGS that can result in direct podocyte injury. Genetic FSGS is increasingly been recognized and a careful evaluation of patients with atypical primary or secondary FSGS should be performed to exclude genetic causes. Unlike primary FSGS, secondary and genetic forms of FSGS do not respond to immunosuppression and tend not to recur after kidney transplantation. Distinguishing primary FSGS from secondary and genetic causes has a prognostic signif-
\end{abstract}

icance and is crucial for an appropriate management. In this review, we examine the pathogenesis, clinical approach to distinguish between the different causes, and current recommendations in the management of FSGS.

(c) 2020 S. Karger AG, Basel

\section{Introduction}

Focal segmental glomerulosclerosis (FSGS) is not a single disease, but rather a histological pattern of glomerular lesion that includes several completely different clinicopathological diseases that share injury within the podocyte as a primary pathophysiological feature [1]. The pattern of glomerular sclerosis is focal at an early stage of the process, affecting a minority of glomeruli, and segmental, involving a section of the glomerular tuft. More diffuse and global glomerulosclerosis occurs as the disease progresses. Cell-to-cell propagation of podocyte injury may explain the characteristic segmental nature of the sclerosing lesions in the glomerular tuft, until the whole glomerulus is affected.

According to the etiology, FSGS lesion has been classified into primary, genetic, and secondary forms that include maladaptive, virus-associated, and medication-induced FSGS. There is a frequent overlap in the clinicopathological features of these different forms, which often poses a diagnosis challenge. Distinguishing between the

$\begin{aligned} & \text { karger@karger.com } \\ & \text { www.karger.com/nef }\end{aligned}$
Karger ${ }^{\prime /}$

Dr. Gema Fernández-Juárez 


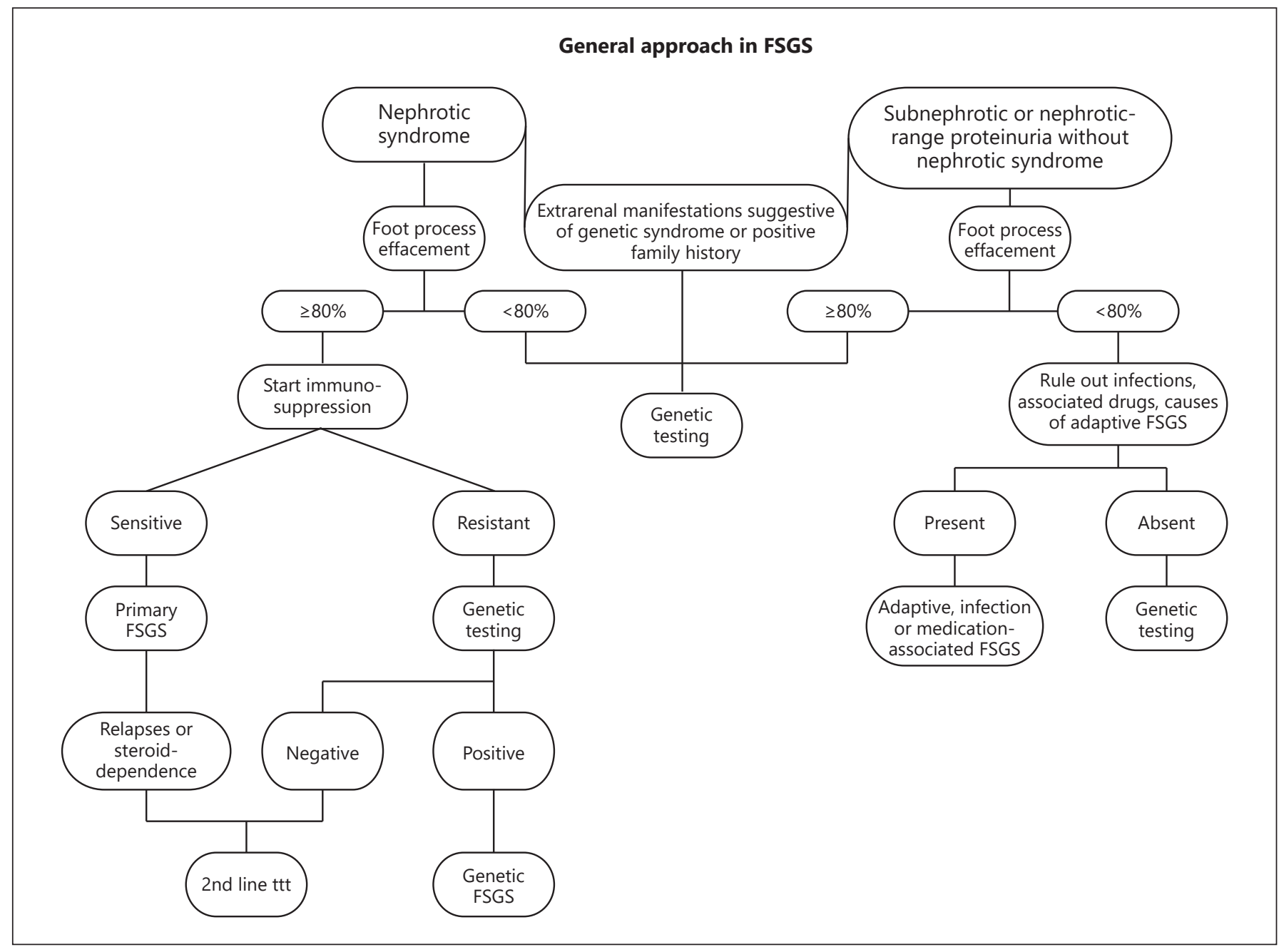

Fig. 1. General approach in FSGS. FSGS, focal segmental glomerulosclerosis.

different forms is crucial since management must be tailored according to the underlying etiology. Primary FSGS is presumably an immunological disease caused by an unknown circulating factor that leads to complete nephrotic syndrome. It may respond to immunosuppressive treatment and tend to recur after kidney transplantation in the first days or weeks [2].

On the other hand, maladaptive and secondary forms often present with subnephrotic proteinuria and progressive kidney insufficiency, do not respond to immunosuppression, and the treatment focuses in resolving the underlying cause. These forms do not recur in kidney grafts, unless the underlying cause that triggered FSGS is not resolved [3].

Genetic causes of FSGS are becoming increasingly recognized, from typical pediatric cases with familiar aggre- gations to sporadic mutations that can be found in early adulthood. Genetic FSGS is typically resistant to immunosuppressive treatment and the transplant relapse rate is low [4]. In this review, we intend to discuss the differences in the pathogenesis, and clinical and pathological aspects of primary, genetic, and secondary forms of FSGS, with the aim of aiding in the choice of the best treatment regimen in each situation, and therefore, improving therapeutic outcomes (Fig. 1,2).

\section{Epidemiology}

The precise incidence and prevalence of FSGS is difficult to establish due to marked geographical and racial differences [5]. The estimated incidence of FSGS varies 


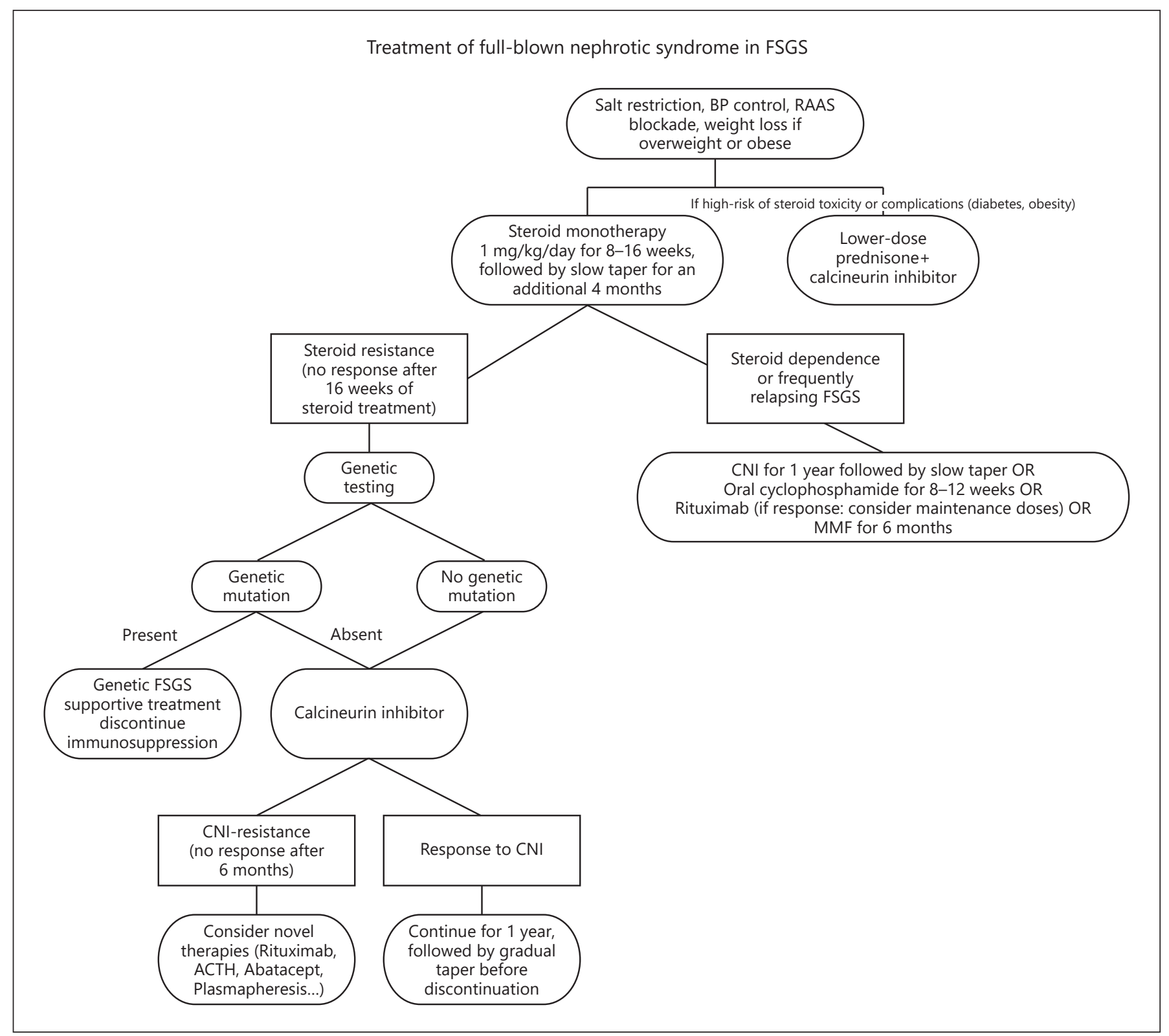

Fig. 2. Treatment of nephrotic syndrome in FSGS. FSGS, focal segmental glomerulosclerosis; CNI, calcineurin inhibitor; MMF, mycophenolate mofetil.

from 1.4 to 21 cases per million population. FSGS can occur at any age, taking place in around $7-10 \%$ of children and $20-30 \%$ of adults with nephrotic syndrome. In adults, FSGS is more common in males, with a 1.5 - to 2 -fold increased incidence compared to females. The incidence of FSGS is around 5 times higher in black patients when compared to white patients, with an annual incidence of 24 cases and 5 cases per million population, respectively, in the USA [6]. This difference is probably due to the in- creased presence of 2 variants of podocyte function proteins in black patients: nonmuscle myosin heavy chain-9 (MYH9) and apolipoprotein L1 (APOL1), which are found in around $60 \%$ of black patients compared to $5 \%$ in white patients [7].

There has been a steady increase in the prevalence of FSGS worldwide over the past few decades, having become the most frequent primary glomerular disease that reaches ESKD in the USA. In a recent clinicopathological 
study that distinguished primary from secondary forms of FSGS, it was observed that although the incidence rate of FSGS is increasing, the proportion between primary and secondary forms has remained stable for the last 20 years [8].

\section{Pathogenesis}

The main site of injury in FSGS is the podocyte. Podocytes are highly differentiated cells that maintain the integrity of glomerular architecture and require a complex cytoskeletal structure in order to function. They may be the target of injury through several ways: nonmechanical insults (immunologic, toxic, and viral), mechanical insults, and genetic disorders [9]. Regardless of the type of stress, injured podocytes are missed into the urinary space, inducing podocyte depletion leaving the glomerular basement membrane uncovered and allowing a direct and potentially harmful interaction between capillary loops and the parietal epithelial cells. Additionally, to balance this lack of podocytes, hypertrophy of the remaining podocytes takes place to cover the glomerular capillary surface. The compensatory intracapillary hypertension results in podocyte and endothelial cell injury, as well as mesangial alterations that lead to progressive focal and segmental sclerosis [10].

The type of damage, the rate of progression, and the severity of podocyte injury varies considerably according to the underlying etiological insult, explaining the differences in clinical and diagnostic features among the distinct forms of FSGS [11]. Primary FSGS causes a rapid and global damage in the podocytes; therefore, the clinical onset is abrupt and podocytopathy is extensive under the electron microscope, showing podocyte foot process effacement in more than $80 \%$ of the capillary surface. Unlike primary FSGS, in secondary or adaptive forms of FSGS, the insult is slow and sustained; therefore, the clinical evolution is progressive and podocyte foot effacement is relatively mild under the electron microscope, at least in early phases.

\section{Primary FSGS}

In primary FSGS, a permeability factor has been described. The nature of this factor has not yet been well identified. It is hypothesized to consist of cytokines that disturb podocyte function and increase glomerular permeability [12]. The best evidence for the presence of a circulating factor comes from the experience with rapid recurrence of FSGS (hours to weeks) after kidney trans- plantation [13]. A remarkable case that corroborates this hypothesis described a case of rapid recurrence of FSGS in a kidney transplant recipient with primary FSGS, developing marked proteinuria, hypoalbuminemia, and declining allograft function, with a biopsy that showed podocyte foot process effacement. The graft was removed and retransplanted into a patient with ESKD due to diabetic nephropathy, showing a reduction in proteinuria, serum $\mathrm{Cr}$, and resolution of foot process effacement in the graft biopsy [14].

Several molecules have been proposed that include apA1b (an isoform of ApoA1), cardiotrophin-like cytokine factor, anti-CD40 antibody, and serum urine-type plasminogen activator receptor (suPAR) [12]. Serum suPAR levels were shown to be elevated in 2 cohorts of children and adults with biopsy-proven FSGS lesions and not in disease controls [15]. However, more recent studies have reported an inverse correlation between estimated glomerular filtration rate (GFR) and suPAR [16], and it is apparent that more studies are needed to clarify its role.

\section{Maladaptive FSGS}

In secondary FSGS forms, most of the cases are maladaptive and result from a mismatch between glomerular load and glomerular capacity. Conditions associated with a reduced renal mass include low nephronal endowment at birth; renal anomalies such as reflux nephropathy; hypoplastic kidney that might be overwhelmed by an increase in total kidney GFR, in conditions such as overweight, obesity, high intake of proteins, or androgen abuse; and AKI [17]. Any chronic glomerular or tubular disease may reduce the total nephron function and result in maladaptive FSGS that is superimposed on the primary disorder.

Maladaptive FSGS arises from the processes described above involving increased single-nephron GFR, leading to a vicious cycle of glomerular hypertrophy, podocyte hypertrophy, stress, and depletion with the eventual formation of synechia and excess extracellular matrix deposition within the glomerulus as was described by Kriz and Lemley [18]. Kidney biopsy features that support the diagnosis of maladaptive FSGS include large glomeruli, a preponderance of perihilar scars among glomeruli showing sclerotic changes, and only partial foot process effacement. Clinical features include normal serum albumin, which is unusual in primary FSGS. Achieving a complete response after treatment with renin-angiotensin system blockade combined with sodium restriction would support a diagnosis of maladaptive FSGS [19]. 
Virus-Induced FSGS

Viruses can induce FSGS by affecting the podocyte, either by direct infection or by release of inflammatory cytokines that engage with podocyte receptors. HIV-1 is the best known viral infection that can cause FSGS, by directly infecting podocytes and tubular epithelial cells. In these cases, even after antiviral therapy and achieving normal serum CD4 levels, podocytes remain reservoirs for HIV-1 [20]. Parvovirus B-19 is another viral agent that can directly infect podocytes and tubular epithelium [21].

\section{Drug-Induced FSGS}

Pamidronate, an osteoclast inhibitor might have direct toxic effects on the actin cytoskeleton of osteoclast. It has been proposed that a similar effect on the podocyte cytoskeleton may take place, inducing FSGS [22]. All forms of interferon may also induce FSGS via a direct podocyte effect, since the podocyte expresses receptors for both interferon $\alpha$ and $\beta$, and express an MHC class II antigen as a response to interferon $\gamma$ [23]. Anabolic steroid abuse has also been associated with the development of FSGS, and it is thought to be due to a combination of a direct nephrotoxic effect and maladaptive glomerular changes secondary to the increased lean body mass [24].

\section{Genetic FSGS}

More than 50 genes have been described as potentially responsible for monogenic forms of FSGS, including those involved in slit diaphragm structure and function, actin cytoskeleton, or cell-signaling apparatus [4, 25]. The relevance of the affected protein in this structure will determine the severity and precocity of the damage. For instance, FSGS caused by NPHS1 or NHPS2 mutations, in which an essential slit diaphragm component nephrin or podocin are absent, results in diffuse foot process effacement with severe congenital nephrotic syndrome [26].

In contrast, other genetic mutations or polymorphisms may simply induce a cytoskeletal disorganization that makes the podocyte more vulnerable. In this position lie mutations in ACTN4 or in structural components of GBM-like type IV collagen genes (COL4A3, COL4A4, and COL4A5). Generally, their onset is usually in late youth or early adulthood, causes a slow progressive damage, and their clinical and pathological characteristics mimic those of secondary FSGS forms. Further environmental, genetic, or epigenetic modifiers could explain the development of distinct phenotypes in family members affected by the same mutation [27, 28].
In addition to mutations in genes encoding podocytespecific proteins, mutations in other genes such as the COQ2 gene, Wilms' tumor gene (WT1), and gene for LIM homeobox transcription factor $1 \beta$ (LMX1B), which is a transcription factor required for the expression of CD2AP and NPHS2, associated with nail-patella syndrome, are associated with syndromes of which FSGS is often a part [4].

\section{APOL1 FSGS}

African Americans have a disproportionate risk for several forms of kidney disease, including a 4 -fold increased risk for FSGS and 18- to 50-fold increased risk for HIVassociated FSGS [7, 29]. Polymorphism in the APOL1 gene associated with the FSGS lesion is usually expressed in individuals of African descent. It has been shown by 2 different groups that 2 variants (G1 and G2) of APOL1 gene are the main factors that induce risk [30,31], with a predilection for collapsing forms of FSGS lesions [32].

APOL1 FSGS is associated with more advanced glomerulosclerosis and interstitial fibrosis at diagnosis and a more rapid progression to ESKD [33]. The direct effect of APOL1, a lipid-binding protein that protects low-density lipoproteins from oxidation, on glomeruli is not known. It has been hypothetized that APOL1 overexpression in glomerular arterioles may provide a first hit by promoting vascular sclerosis, and that a second hit, which could be environmental, infectious, or genetic, would be needed for the expression of disease [34].

\section{Differential Diagnosis}

Discrimination between primary, genetic, and secondary forms should be assessed by the presence or absence of the following clinical or histological variables: (1) complete nephrotic syndrome (primary FSGS), (2) subnephrotic or nephrotic-range proteinuria with normal serum albumin (secondary FSGS), (3) the presence of known causes of secondary FSGS, (4) family history (genetic FSGS), and (5)characteristics of podocyte foot effacement in electron microscopy. Genetic FSGS forms are specially challenging because they show variable clinical and pathological features and could mimic either primary or secondary forms [3]. There is consensus on performing genetic tests in steroid-resistant FSGS in patients with positive family history or extrarenal physical signs suggestive of a genetic disorder (Fabry disease, nail-patella syndrome, or Alport syndrome) [4, 25, 28, 35]. Some studies have suggested conducting a genetic study in pa- 


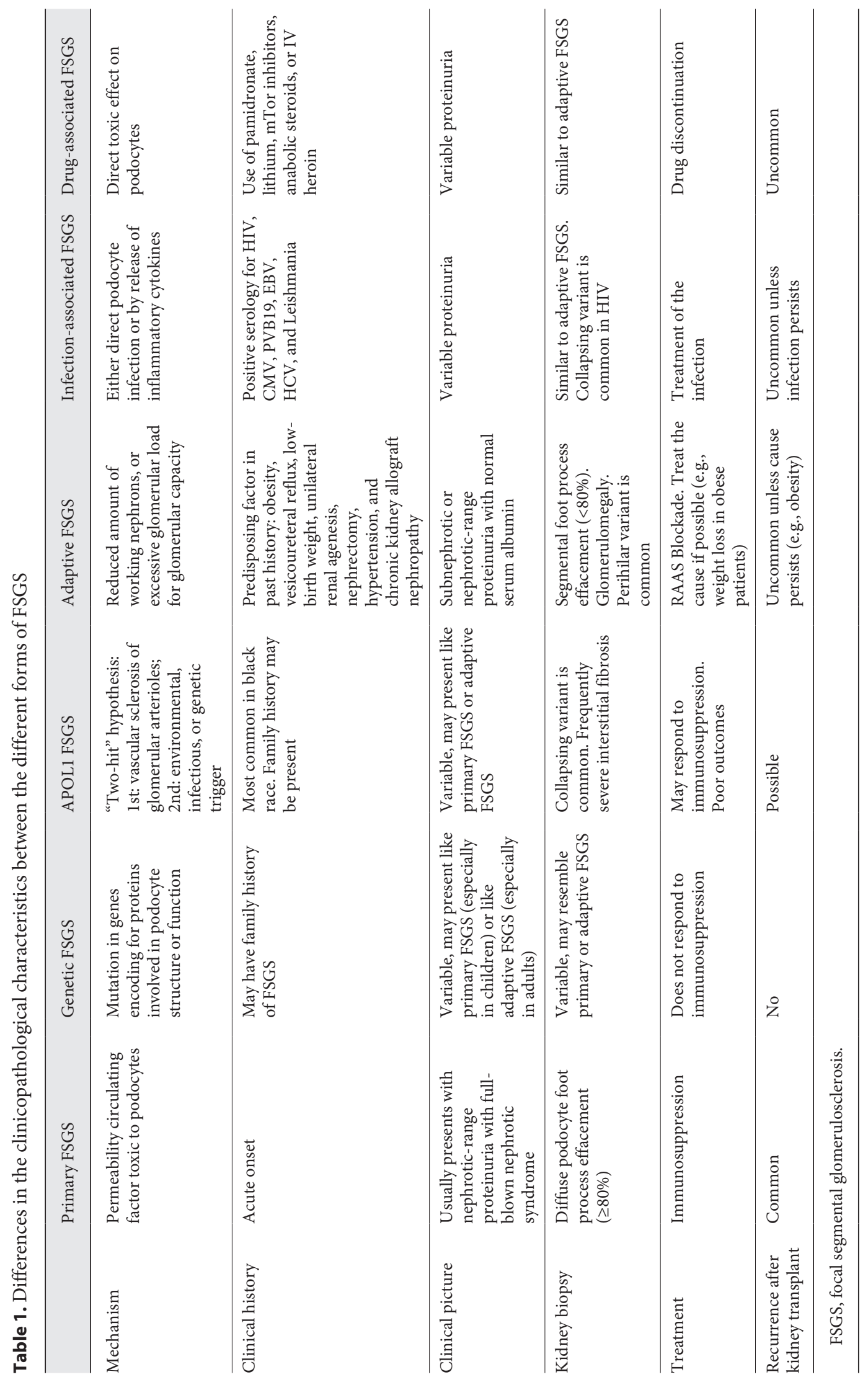


Table 2. Risk factors for progression to ESKD in FSGS

\begin{tabular}{|c|c|c|}
\hline Clinical features at biopsy & Clinical features during disease course & Histopathologic features at biopsy \\
\hline 1. Severity of nephrotic-range proteinuria & $\begin{array}{l}\text { 1. Failure to achieve partial or complete } \\
\text { remission }\end{array}$ & 1. Collapsing variant \\
\hline $\begin{array}{l}\text { 2. Elevated serum } \mathrm{Cr}(>1.3 \mathrm{mg} / \mathrm{dL}) \text { at presentation } \\
\text { 3. Black race }\end{array}$ & 2. Uncontrolled hypertension & $\begin{array}{l}\text { 2. Tubulointerstitial fibrosis } \\
\text { 3. Mesangial IgM or C3 deposits }\end{array}$ \\
\hline
\end{tabular}

FSGS, focal segmental glomerulosclerosis.

Table 3. Histologic variants of FSGS according to the Columbia Classification

\begin{tabular}{|c|c|c|c|}
\hline Variant & Histologic feature & Clinical features/prognosis & $\begin{array}{l}\text { Progression } \\
\text { to ESKD, \% }\end{array}$ \\
\hline Collapsing & $\begin{array}{l}\text { Podocyte hyperplasia collapsing } \\
\text { glomerular tuft }\end{array}$ & $\begin{array}{l}\text { Worst prognosis. Usually presents with abrupt onset of severe } \\
\text { nephrotic syndrome. Predominant in black race. Poor response to } \\
\text { steroids }\end{array}$ & $\sim 70$ \\
\hline Cellular & $\begin{array}{l}\text { Podocyte hyperplasia, endocapillary } \\
\text { proliferation. Usually severe foot } \\
\text { process effacement }\end{array}$ & $\begin{array}{l}\text { Early stage in the evolution of FSGS. Least common variant. } \\
\text { Usually primary FSGS, but could be seen in other forms of FSGS }\end{array}$ & $\sim 30$ \\
\hline Perihilar & $\begin{array}{l}\text { Segmental sclerosis at the vascular } \\
\text { pole }\end{array}$ & $\begin{array}{l}\text { Most common in adaptive FSGS. Usually presents with } \\
\text { subnephrotic proteinuria or nephrotic-range proteinuria and } \\
\text { normal serum albumin }\end{array}$ & $30-50$ \\
\hline Tip lesion & $\begin{array}{l}\text { Segmental sclerosis at the proximal } \\
\text { tubular pole }\end{array}$ & $\begin{array}{l}\text { Best prognosis. Usually primary. Most common in white patients, } \\
\text { frequently responds to steroids. May present reversible AKI at } \\
\text { presentation }\end{array}$ & $5-20$ \\
\hline $\begin{array}{l}\text { Classic } \\
\text { (NOS) }\end{array}$ & $\begin{array}{l}\text { Segmental sclerosis, not meeting the } \\
\text { definition of the other variants }\end{array}$ & Most common subtype, could be found in any form of FSGS & $30-40$ \\
\hline
\end{tabular}

tients with clinical characteristics of secondary FSGS when a secondary cause has been carefully ruled out (including overweight) [3]. A recent study has reported that extended genetic testing followed by reverse phenotyping increases the diagnostic precision in steroid-resistant nephrotic syndrome [36]. Table 1 summarizes the main differences between the different forms of FSGS (Table 2).

\section{Pathological Features and Histologic Variants}

FSGS is classified according to the Columbia Classification into 5 variants [37]. The different histologic variants of FSGS show different clinical presentations and outcomes, which are summarized in Table 3.

This classification can be applied to primary, genetic, and secondary forms of FSGS and might be a useful prog- nostic indicator in FSGS. However, caution should be applied when using the classification to guide in management decisions, given the inherent limitations of biopsy sampling and variability in applying the criterion for each subtype. The histologic variant does not predict risk of recurrence in renal allografts, and interestingly, different variants can be seen before and after transplantation in the same patient [13].

Distinguishing minimal change disease (MCD) from FSGS is essential since both entities present with proteinuria, share podocyte injury as the main target of lesion, and show scarce immune deposits, if any, on direct immunofluorescence. An adequate renal cortical sample is needed to exclude FSGS, and simulation studies have shown that if glomerular scars are regularly distributed in a biopsy with 10-30 glomeruli, the diagnostic accuracy for detecting at least one scarred glomerulus will be $80 \%$ 
Table 4. Risk factors for recurrence of FSGS in kidney transplant recipients
Risk factors for FSGS recurrence after kidney transplantation

1. Childhood onset of the initial disease (particularly between 6 and 15 years)

2. Rapid progression of primary FSGS to ESKD ( $<3$ years)

3. Initial steroid sensitivity in children with primary FSGS

4. White race recipient of African American donor

5. Loss of previous allografts due to recurrent FSGS

6. Patients receiving live donor grafts

7. Severe hypoalbuminemia at presentation $(<2.5 \mathrm{~g} / \mathrm{dL})$

8. Absent family history of FSGS

FSGS, focal segmental glomerulosclerosis. when at least $10 \%$ of juxtamedullary glomeruli or $20 \%$ of other cortical glomeruli are scarred, and with an average of 20 glomeruli with $20-60 \%$ glomerular sclerosis, the predicted error rate is $\pm 50 \%$ for extent of glomerular involvement [38]. This suggests caution when making treatment recommendations on the basis of the extent of involved glomeruli.

Although electronic microscopy is essential in any kidney biopsy assessment, in FSGS lesion, it is fundamental to distinguish between the different etiologies [3]. First of all, the severity of podocyte foot process effacement will guide into diagnosing primary or secondary causes of FSGS since foot process effacement is greater in primary FSGS (usually $>80 \%$ of basement membrane's surface area) compared to maladaptive FSGS which shows more irregular and patchy effacement. It can also rule out basement membrane anomalies or deposits that could be seen in genetic disorders such as Fabry disease and type IV collagen diseases, and it can also exclude the presence of immune deposits and fibrils [11].

\section{FSGS in Kidney Transplants}

Following kidney transplantation, recurrent or de novo FSGS may develop in the kidney allograft and may be primary (idiopathic) or secondary to hyperfiltration, infection, medication, and rarely due to genetic mutation. According to the Australian and New Zealand Dialysis and Transplant registry, patients with histology evidence of FSGS receiving kidney grafts have a significantly inferior 5 -year graft survival compared to graft recipients with any other cause of kidney disease ( 81 vs. $88 \%$ ). This difference is more pronounced in the pediatric population, where the 5-year graft survival in recipients with FSGS was $68 \%$ compared to $93 \%$ in recipients with any other cause of kidney disease. FSGS patients receiving live donor grafts had a significantly increased graft survival (14.8 years) compared to recipients of deceased donor grafts (12.1 years), although there was a 2 -fold risk of disease recurrence in patients receiving live donor grafts [39].

\section{Recurrent FSGS}

FSGS commonly recurs in kidney grafts. The reported recurrence rate varies widely from 4 to $66 \%$ across the different series. These studies include cases of FSGS due to hyperfiltration and other maladaptive or secondary forms, which are much less likely to recur in the kidney allograft than primary FSGS. Therefore, the recurrence rate of primary FSGS alone is probably much higher. FSGS recurrence can be sudden, with appearance of nephrotic-range proteinuria within the first days or even hours after transplantation. The main risk factors for recurrence include childhood onset of the initial disease, the rapid progression to ESKD, a history of recurrence in a previous allograft, Caucasian recipients from African American donors, live donor grafts, severe hypoalbuminemia at presentation, and the absence of a positive family history for FSGS [40, 41] (Table 4). Initial steroid sensitivity has also been associated with recurrence after kidney transplantation in children [42]. Recurrence is most common in the first 2 years posttransplantation, and it is a strong predictor for graft outcome, leading to graft loss in $52 \%$ of the patients within 5 years, compared to $83 \%$ survival in those who do not recur [39]. Most grafts are lost within the first 2 years after recurrence. The best treatment for recurrent FSGS is not known. Prophylactic plasmapheresis does not reduce the risk for recurrence, but therapeutic plasmapheresis is still considered a good option as first-line treatment, especially in early recurrences with severe proteinuria [43]. Small studies have suggested pre-emptive treatment with rituximab to prevent recur- 
rence, but more recent studies have shown that it is not effective to reduce the risk of recurrence [44].

\section{De Novo FSGS}

It is the most common type (up to 20\%) of de novo glomerulonephritis after transplantation [45]. Several factors may predispose the development of de novo FSGS after kidney transplantation, predominantly those that result in compensatory hyperfiltration associated with a reduced nephron mass such as in diabetes, hypertension, BK infection, calcineurin inhibitor (CNI) therapy, and rejection [46]. The introduction of sirolimus can induce de novo FSGS through a direct effect on podocyte integrity [47]. Contrary to recurrent FSGS, most cases of de novo FSGS develop at later stages after transplantation. De novo FSGS has a poor prognosis, with a $40 \%$ allograft survival after 5 years of diagnosis [48]. The mainstay of treatment revolves around removal or treatment of the causative agent and anti-proteinuric treatment.

\section{Treatment}

Therapy should be tailored based on the etiological form of FSGS, avoiding immunosuppressive treatment in non-primary FSGS. We should also consider other individual factors such as age, GFR, and comorbidities [11].

In patients with secondary or genetic FSGS, the approach consists of dietary salt restriction, strict blood pressure control, weight loss for those who are overweight or obese, and renin-angiotensin system blockade with either angiotensin-converting enzyme inhibitors or angiotensin receptor blockers [49]. In FSGS induced by a drug or infection, treatment should be directed towards the cause, discontinuing the drug if possible or treating the infection.

Although treatment with the general measures mentioned before should be started in all patients, in cases with full-blown nephrotic syndrome, immunosuppressive therapy should be started immediately since these patients have a poor prognosis without disease-modifying therapy [50]. There are no randomized trials comparing prednisone or other agents with placebo for initial therapy of primary FSGS, and the recommendations are given following data from observational studies.

It remains unclear whether the benefit of immunosuppressants relies solely on their immunosuppressive effect, or whether it is due to a local effect on podocyte function. Rituximab has been shown to directly affect podocyte function in an SMPDL-3b-dependent manner in recur-

Update in Focal Segmental

Glomerulosclerosis rent FSGS [51], while it has been shown that the effect of cyclosporine (CsA) on proteinuria is not dependent on NFAT inhibition in T cells but rather results from its effects on kidney podocytes, including the stabilization of synaptopodin [52].

Prophylactic anticoagulation has not specifically been studied in FSGS, and treatment with anticoagulants in FSGS with nephrotic syndrome is still controversial [53]. It might be considered in patients with very severe nephrotic syndrome.

\section{Initial Immunosuppression: Corticosteroids}

In adults with primary FSGS and overt nephrotic syndrome, the initial immunosuppressive therapy consists of high-dose steroids alone [50,54]. The optimal dose and duration of prednisone treatment is controversial. The standard regimen could be oral prednisone at a dose of $1 \mathrm{mg} / \mathrm{kg}$ /day (maximum dose 60-80 mg/day) for 2-4 months, followed by a tapered dose reduction for an additional 4 months $[55,56]$. With this regimen, proteinuria usually decreases within the first 2 months but could take up to 16 weeks for remission. In general, the duration of therapy will vary according to the degree and rapidity of response. Shorter courses ( $<2$ months) result in much lower remission rates $(20-30 \%)$ and may lead to the wrong belief that the patient was not steroid responsive [1]. The extended regimen of steroids compared to treatment of MCD is necessary to achieve remission [57].

In an attempt to minimize the potential toxicity of long-term high-dose daily steroid, a regimen with alternate-day high-dose steroids (120 mg on alternate days) could be of choice. A study that assessed this regimen in patients with histologic evidence of FSGS and $>60$ years, a complete remission rate of $44 \%$ was achieved [58].

The initial response of proteinuria to corticosteroids is the strongest prognostic indicator of renal survival, regardless of the histologic variant $[1,55,57]$. Patients who undergo complete or partial remission $(40-80 \%$ of patients) have better chance of renal survival. Conversely, those patients who have little or no reduction in proteinuria after 12-16 weeks of prednisone should be considered corticosteroid resistant and have the worst renal prognosis. Additionally, patients who relapse when prednisone is being tapered or immediately after prednisone treatment is over are called corticosteroid dependent and carry an intermediate prognosis regarding renal survival.

It has been suggested that an initial immunosuppressive regimen with CsA combined with low-dose prednisone could be given in patients with higher risk of steroid toxicity, such as in patients with diabetes mellitus or se- 
vere osteoporosis. In a retrospective study that compared patients receiving oral prednisone alone $(1 \mathrm{mg} / \mathrm{kg} /$ day $)$ for 4 months, or low-dose prednisone $(0.5 \mathrm{mg} / \mathrm{kg} / \mathrm{day})$ combined with CsA or azathioprine, there were no differences in remission between the 3 groups [59]. In another retrospective study, CNIs), with or without steroids, were noninferior to treatment with steroid monotherapy, and all treatment groups showed improved renal survival compared to supportive treatment [60]. CNIs should be avoided in patients who have significantly reduced kidney function (eGFR $<30 \mathrm{~mL} / \mathrm{min} / 1.73 \mathrm{~m}^{2}$ ) due to the potential nephrotoxicity of these drugs. In this situation, we can consider the use of mycophenolate mofetil (MMF). A study randomized patients with histologic evidence of FSGS to receive MMF $1 \mathrm{~g}$ twice daily for 6 months plus prednisone at a dose of $0.5 \mathrm{mg} / \mathrm{kg} / \mathrm{day}$ for 2-3 months compared to the standard prednisone monotherapy [61]. There were no differences in remission and relapse rates, with less steroid exposure in the MMF group. However, it must be noted that this study did not distinguish between primary, genetic, or secondary forms of FSGS.

\section{Treatment in Relapsing FSGS}

Relapse can occur months after achieving complete remission (33\%) or after achieving partial remission (50\%). Patients who relapse have a poorer renal survival than those who do not, but they still have a better prognosis than those who never achieve remission $[55,62]$.

In patients who had an acceptable tolerance to the previous glucocorticosteroid regimen, a repeat course with a slower taper over a longer period of time is the first choice. Otherwise, if the patient had significant glucocorticosteroid-induced toxicity during the initial therapy, or has had multiple relapses, we could use a CNI-based regimen (or MMF if eGFR $<30 \mathrm{~mL} / \mathrm{min} / 1.73 \mathrm{~m}^{2}$ ) with or without low-dose of prednisone [50].

\section{Treatment in Corticosteroid-Resistant FSGS}

Recommendations for therapy in steroid-dependent and steroid-resistant FSGS are based upon a few randomized controlled trials. The first option in these cases is treatment with CNI with or without steroids. MMF and cytotoxic agents remain as second-line options. In cases where these options fail to achieve remission, novel therapies should be considered.

In steroid-resistant FSGS in adult patients, CsA has shown to be the most effective agent to induce a remission. Two randomized controlled trials have shown the benefit of treatment with CsA in adult steroid-resistant
FSGS; the first study showed that CsA $(6 \mathrm{mg} / \mathrm{kg} /$ day in children and $5 \mathrm{mg} / \mathrm{kg} /$ day in adults) for 6 months without steroids followed by a dose taper for 2 months until discontinuation induced more remission (complete remission in $21 \%$ of patients and partial remission in $36 \%)$ compared to supportive therapy [63]. The second study showed that CsA at an initial dose of $3.5 \mathrm{mg} / \mathrm{kg} /$ day titrated according to trough levels, with low-dose prednisone $(0.15 \mathrm{mg} / \mathrm{kg} /$ day $)$ for 6 months with a 1 -month taper achieved more remission (12\% complete and $57 \%$ partial) compared to placebo [64]. However, the relapse rate after 1 year was of 75 and $60 \%$ of the patients in the respective studies. In spite of relapses, renal survival was better in patients with CsA for 6 months than in placebo.

Following the results of these 2 randomized clinical trials, the standard first-line therapy for steroid-resistant FSGS is considered to be CsA for 6 months, with or without steroids. But taking into notice the high rate of relapses, we recommend that if partial or complete remission is achieved, CsA should be continued for at least one more year, and very gradually tapered before discontinuation. To minimize potential CsA nephrotoxicity and maintain remission, it should be slowly tapered by 0.5 $\mathrm{mg} / \mathrm{kg} / \mathrm{month}$ to the lowest effective dose. On the other hand, if no remission is achieved after a 6-month regimen, it should be discontinued and an alternative therapy should be considered.

The use of another CNI, tacrolimus, has shown improved remission rates by $15 \%$ in CsA-dependent or CsA-resistant FSGS, but the relapse rate remained very high (76\% after tacrolimus discontinuation) [65]. In another clinical trial, corticosteroid-resistant patients were randomized to CsA or MMF with alternate-day prednisone plus pulse dexamethasone, with no differences in complete or partial remission rates between both groups [66].

In steroid-resistant and CsA-resistant FSGS, MMF has been assessed at a dose of $1 \mathrm{~g}$ twice daily for 6 months. In one study, no patient achieved complete remission, and $44 \%$ of patients achieved partial remission [67]. Another study evaluated MMF in resistant patients, with a remission rate of $54 \%$, showing that it may be of use in resistant cases of FSGS [48]. MMF is the choice option when GFR is below $30 \mathrm{~mL} / \mathrm{min} / 1.73 \mathrm{~m}^{2}$.

Rituximab has been increasingly used for steroid-resistant FSGS [69], and there are several small retrospective observational studies that have shown the safety and efficacy of rituximab in children, achieving remission rates up to $41.5 \%$ [70]. However, most of these observa- 
tional studies widely differ in the severity of cases, as well as in the number and dose of rituximab infusions, concomitant immunosuppressive agents, and follow-up period. We believe that an effort should be exerted to standardize rituximab dose and propose prospective studies to evaluate the real efficacy of rituximab in steroid-resistant FSGS.

There are limited data for the use of cytotoxic agents in steroid-resistant FSGS, apart from a few retrospective studies. A randomized trial that compared chlorambucil with CsA in patients with steroid-resistant FSGS showed no benefit for chlorambucil in this group of patients [71]. We consider that cytotoxic agents have no value in the treatment of steroid-resistant FSGS.

Steroid resistance should raise the alarm of the possibility of an underlying genetic disease. We might find a causal mutation in $43 \%$ of the adults with familiar background and in $10 \%$ of sporadic adult cases with steroidresistant nephrotic syndrome [25]. In children, this rate is higher; mutations were found in $49.7 \%$ of infantile onset cases (4-12 months), $25.3 \%$ in early childhood (13 months -6 years), $17.8 \%$ in late childhood (6-12 years), and $10.8 \%$ in adolescents [72]. Therefore, genetic testing should be performed in steroid-resistant patients regardless of their familiar background or age.

\section{Treatment of Steroid-Dependent or Frequently \\ Relapsing FSGS}

In frequently relapsing steroid-sensitive nephrotic syndrome, a study compared oral cyclophosphamide at a dose $2.5 \mathrm{mg} / \mathrm{kg} /$ day for 2 months with CsA at a dose $5-6 \mathrm{mg} / \mathrm{kg} /$ day in 2 divided doses for 9 months, with similar relapse rates after 9 months, but with significantly less relapses after 2 years of follow-up (37\% in cyclophosphamide group vs. 75\% in CsA group) [73]. Thus, in frequently relapsing FSGS, treatment with CsA should be maintained for a longer duration, with the risk of potential nephrotoxicity. A trial that compared CsA started at a dose $5 \mathrm{mg} / \mathrm{kg} /$ day and adjusting according to trough levels, with chlorambucil at a dose $0.1-0.4 \mathrm{mg} /$ $\mathrm{kg} /$ day for 6-12 weeks, followed by CsA if there was no response, showed no differences in complete or partial remission or renal survival after 4 years of follow-up, but all patients that received chlorambucil required CsA [71]. Therefore, chlorambucil is considered to be of no benefit in steroid-resistant FSGS. There are no data to support the use of cytotoxic drugs in steroid-dependent or steroid-resistant FSGS apart from the previously mentioned trials and small retrospective observational studies.

Update in Focal Segmental

Glomerulosclerosis
There have been several case reports that have described a successful use of rituximab in steroid-dependent FSGS in adults. Five patients with steroid-dependent FSGS achieved complete remission for 10-15 months after 4 doses of weekly rituximab at a dose of $375 \mathrm{mg} / \mathrm{m}^{2}$, with discontinuation of the other immunosuppressants used [74]. On the other hand, no complete remissions were achieved in other patients with steroid-resistant FSGS after treatment with rituximab $[69,75]$. A prospective study assessed the rate of recurrence in children and adults with frequently relapsing MCD and FSGS after rituximab, and after 1 year of follow-up, all patients were in remission and half of them did not recur. Renal function improved, especially in FSGS, and relapse rate was reduced by 5 -fold [76]. This promising result is now modifying the approach to cases of steroid dependence and even considering rituximab for initial treatment of FSGS in some centers. Ofatumumab recognizes a distinct membrane epitope in the human CD20 molecule from rituximab's target, allowing tighter binding between the antibody and the B-cell membrane antigen, achieving higher efficacy in depleting B cells. Ofatumumab was studied in 2 small cohorts of children with nephrotic syndrome resistant to all immunosuppressive treatment, showing that it might induce remission [77]. Currently, 2 clinical trials are ongoing with these drugs (NCT03298698 and NCT02394119).

ACTH therapy may be of benefit in some patients with steroid-dependent or steroid-resistant FSGS. In a small study that included 24 patients, most of which were steroid-resistant FSGS patients, treatment with subcutaneous ACTH gel ( 80 units twice weekly) had limited benefit; 7 patients achieved partial or complete remission after a mean 16 months of follow-up, but 2 out of them relapsed [78]. The cost of ACTH gel limits the ability to conduct randomized trials to confirm these findings.

\section{Novel Therapies}

Abatacept inhibits T-cell costimulatory signals mediated by CD80 (B7-1), which is hypothesized that it may contribute in the pathogenesis of the disease. In 4 patients with relapsing posttransplant FSGS and 1 patient with steroid-resistant FSGS, abatacept at a dose of $10 \mathrm{mg} / \mathrm{kg}$ (from single to monthly doses) achieved complete remission in 3 patients and partial remission in the other 2 . However, these patients were also treated with plasma exchange and immunosuppression [79]. On the other hand, abatacept was ineffective in 10 patients with posttransplant recurrent FSGS $[80,81]$. To be able to recommend 
abatacept in primary FSGS, further studies are required to confirm their benefit.

Sparsentan is a selective antagonist of the angiotensin II receptor and the endothelial A receptor. A phase 2, randomized, double-blind trial evaluated the efficacy and safety of sparsentan in patients with histologic evidence of FSGS. Patients were randomly assigned to receive sparsentan at different doses or irbesartan (300 mg/day) for 8 weeks, showing a greater reduction in proteinuria among sparsentan-treated patients compared with irbesartan-treated patients, with no significant differences between each sparsentan dose groups and irbesartan. More sparsentan-treated patients (28 vs. $9 \%$ ) achieved a partial remission compared to irbesartan, with no significant differences in kidney function or adverse events [82]. However, it should be noted that this study included patients with biopsy-proven FSGS lesions without distinguishing between primary, genetic, or secondary forms. Although the authors reported to exclude secondary causes, most patients had non-nephrotic proteinuria; therefore, the studied cohorts likely included mixed forms of FSGS. More specific information may come from a randomized, multicenter, double-blind, parallel, phase 3 trial (NCT03493685), designed to study the nephroprotective effect of sparsentan compared to irbesartan in patients with primary and genetic FSGS.

Bleselumab is currently being studied as part of the immunosuppressive regimen after kidney transplantation instead of MMF to assess its protective role in recurrent FSGS, in a phase 2, multicenter, randomized, open-label trial (NCT02921789) during a year follow-up. Other drugs such as Fresolimumab (NCT01665391) are now under evaluation in phase 2 clinical trials for treatment of steroid-resistant FSGS.

\section{Plasmapheresis}

There is a limited role for plasmapheresis in the treatment of primary FSGS, based upon studies in patients with recurrent FSGS after kidney transplantation. In these cases, removal of a circulating factor by a protein column adsorption or plasma exchange can reduce proteinuria and sometimes induce complete remission. This effect may be temporary since proteinuria recurs to previous levels within 2 months of treatment. In a study in patients with treatment-resistant primary FSGS, plasmapheresis showed benefit in some patients after a mean 17 sessions, with complete remission or partial remission in $73 \%$ of patients, achieving stable kidney function [83]. On the other hand, another study showed poorer results, with only $25 \%$ of partial remission after plasmapheresis
[84]. Following these results, some centers routinely prescribe plasma exchange therapy in all patients with steroid-resistant FSGS, along with immunosuppressive therapy.

Several small studies have assessed the role of low-density lipoprotein apheresis in patients with steroid-resistant primary FSGS, showing a certain reduction in proteinuria and improvement of serum albumin in the short term [85]. The mechanism of this effect is not known yet, and it is hypothesized that the reduction in inflammatory cytokines might be behind this effect.

\section{Conclusion}

FSGS is a common histologic pattern of glomerular injury which is reached through diverse pathological diseases. All forms of FSGS have podocyte injury as the main mediator of the damage, although a completely different pathway is involved in each disease. Clinicians should exert an effort to label each patient properly, avoiding immunosuppresive treatment in non-primary FSGS.

Corticosteroids are still the cornerstone for the treatment of primary FSGS. In steroid-resistant or dependent patients, CNI is the option of choice, but their relapse rate is very high, and a very slow taper is recommended. MMF might be considered when renal function is not preserved $\left(\mathrm{GFR}<30 \mathrm{~mL} / \mathrm{min} / 1.73 \mathrm{~m}^{2}\right)$. Currently, new corticosteroid-sparing agents are being studied. Anti-CD20 monoclonal antibodies might modify the approach to cases of steroid dependence. We need a wider understanding of the mechanism of the disease in order to improve our skills to identify the key pathogenetic target and, thus, uncover new targeted therapies in the future to offer a tailored treatment that could improve renal outcomes in FSGS.

\section{Conflict of Interest Statement}

The authors have no conflicts of interest to declare.

\section{Funding Sources}

The authors did not receive any funding. 


\section{References}

1 Rydel JJ, Korbet SM, Borok RZ, Schwartz MM. Focal segmental glomerular sclerosis in adults: presentation, course, and response to treatment. Am J Kidney Dis. 1995 Apr;25(4): $534-42$.

2 Schwartz MM, Korbet SM. Primary focal segmental glomerulosclerosis: pathology, histological variants, and pathogenesis. Am J Kidney Dis. 1993 Dec;22(6):874-83.

3 De Vriese AS, Sethi S, Nath KA, Glassock RJ, Fervenza FC. Differentiating primary, genetic, and secondary FSGS in adults: a clinicopathologic approach. J Am Soc Nephrol. 2018;29(3):759-74.

4 Rood IM, Deegens JK, Wetzels JF. Genetic causes of focal segmental glomerulosclerosis: implications for clinical practice. Nephrol Dial Transplant. 2012 Mar;27(3):882-90.

5 Sim JJ, Batech M, Hever A, Harrison TN, Avelar T, Kanter MH, et al. Distribution of biopsy-proven presumed primary glomerulonephropathies in 2000-2011 among a racially and ethnically diverse US population. Am J Kidney Dis. 2016 Oct;68(4):533-44.

6 Kitiyakara C, Kopp JB, Eggers P. Trends in the epidemiology of focal segmental glomerulosclerosis. Semin Nephrol. 2003 Mar;23(2): $172-82$.

7 Parsa A, Kao WH, Xie D, Astor BC, Li M, Hsu $\mathrm{CY}$, et al. APOL1 risk variants, race, and progression of chronic kidney disease. N Engl J Med. 2013 Dec 5;369(23):2183-96.

8 Hommos MS, De Vriese AS, Alexander MP, Sethi S, Vaughan L, Zand L, et al. The incidence of primary vs secondary focal segmental glomerulosclerosis: a clinicopathologic study. Mayo Clin Proc. 2017 Dec;92(12):1772-81.

9 Kriz W, Elger M, Nagata M, Kretzler M, Uiker S, Koeppen-Hageman I, et al. The role of podocytes in the development of glomerular sclerosis. Kidney Int Suppl. 1994 Feb;45:S6472.

10 Kriz W, Endlich K. Hypertrophy of podocytes: a mechanism to cope with increased glomerular capillary pressures? Kidney Int. 2005 Jan;67(1):373-4.

11 Sethi S, Glassock RJ, Fervenza FC. Focal segmental glomerulosclerosis: towards a better understanding for the practicing nephrologist. Nephrol Dial Transplant. 2015 Mar; 30(3):375-84

12 Königshausen E, Sellin L. Circulating permeability factors in primary focal segmental glomerulosclerosis: a review of proposed candidates. Biomed Res Int. 2016;2016:3765608.

13 Canaud G, Dion D, Zuber J, Gubler MC, Sberro R, Thervet E, et al. Recurrence of nephrotic syndrome after transplantation in a mixed population of children and adults: course of glomerular lesions and value of the Columbia classification of histological variants of focal and segmental glomerulosclerosis (FSGS). Nephrol Dial Transplant. 2010 Apr;25(4): $1321-8$.
14 Gallon L, Leventhal J, Skaro A, Kanwar Y, Alvarado A. Resolution of recurrent focal segmental glomerulosclerosis after retransplantation. N Engl J Med. 2012 Apr 26;366(17): 1648-9.

15 Wei C, Trachtman H, Li J, Dong C, Friedman $\mathrm{AL}$, Gassman JJ, et al. Circulating suPAR in two cohorts of primary FSGS. J Am Soc Nephrol. 2012 Dec;23(12):2051-9.

16 Reiser J, Nast CC, Alachkar N. Permeability factors in focal and segmental glomerulosclerosis. Adv Chronic Kidney Dis. 2014 Sep; 21(5):417-21.

17 Rennke HG, Klein PS. Pathogenesis and significance of nonprimary focal and segmental glomerulosclerosis. Am J Kidney Dis. 1989 Jun;13(6):443-56.

$18 \mathrm{Kriz} \mathrm{W,} \mathrm{Lemley} \mathrm{KV.} \mathrm{A} \mathrm{potential} \mathrm{role} \mathrm{for} \mathrm{me-}$ chanical forces in the detachment of podocytes and the progression of CKD. J Am Soc Nephrol. 2015 Feb;26(2):258-69.

19 Rosenberg AZ, Kopp JB. Focal segmental glomerulosclerosis. Clin J Am Soc Nephrol. 2017 Mar 7;12(3):502-17.

20 Chen P, Chen BK, Mosoian A, Hays T, Ross MJ, Klotman PE, et al. Virological synapses allow HIV-1 uptake and gene expression in renal tubular epithelial cells. J Am Soc Nephrol. 2011 Mar;22(3):496-507.

21 Moudgil A, Nast CC, Bagga A, Wei L, Nurmamet A, Cohen $\mathrm{AH}$, et al. Association of parvovirus B19 infection with idiopathic collapsing glomerulopathy. Kidney Int. 2001 Jun;59(6):2126-33.

22 Markowitz GS, Appel GB, Fine PL, Fenves AZ, Loon NR, Jagannath S, et al. Collapsing focal segmental glomerulosclerosis following treatment with high-dose pamidronate. J Am Soc Nephrol. 2001 Jun;12(6):1164-72.

23 Markowitz GS, Nasr SH, Stokes MB, D'Agati VD. Treatment with IFN- $\alpha,-\beta$, or $-\gamma$ is associated with collapsing focal segmental glomerulosclerosis. Clin J Am Soc Nephrol. 2010 Apr; 5(4):607-15.

24 Herlitz LC, Markowitz GS, Farris AB, Schwimmer JA, Stokes MB, Kunis C, et al. Development of focal segmental glomerulosclerosis after anabolic steroid abuse. J Am Soc Nephrol. 2010 Jan;21(1):163-72.

25 Santín S, Bullich G, Tazón-Vega B, GarcíaMaset R, Giménez I, Silva I, et al. Clinical utility of genetic testing in children and adults with steroid-resistant nephrotic syndrome. Clin J Am Soc Nephrol. 2011 May;6(5):113948.

26 Patrakka J, Kestilä M, Wartiovaara J, Ruotsalainen V, Tissari P, Lenkkeri U, et al. Congenital nephrotic syndrome (NPHS1): features resulting from different mutations in Finnish patients. Kidney Int. 2000 Sep; 58(3):972-80
27 Choi HJ, Lee BH, Cho HY, Moon KC, Ha IS, Nagata M, et al. Familial focal segmental glomerulosclerosis associated with an ACTN4 mutation and paternal germline mosaicism. Am J Kidney Dis. 2008 May;51(5):834-8.

28 Lepori N, Zand L, Sethi S, Fernandez-Juarez G, Fervenza FC. Clinical and pathological phenotype of genetic causes of focal segmental glomerulosclerosis in adults. Clin Kidney J. 2018 Apr;11(2):179-90.

29 Eggers PW, Kimmel PL. Is there an epidemic of HIV Infection in the US ESRD program? J Am Soc Nephrol. 2004 Sep;15(9):2477-85.

30 Tzur S, Rosset S, Shemer R, Yudkovsky G, Selig S, Tarekegn A, et al. Missense mutations in the APOL1 gene are highly associated with end stage kidney disease risk previously attributed to the MYH9 gene. Hum Genet. 2010 Sep;128(3):345-50.

31 Genovese G, Tonna SJ, Knob AU, Appel GB, Katz A, Bernhardy AJ, et al. A risk allele for focal segmental glomerulosclerosis in African Americans is located within a region containing APOL1 and MYH9. Kidney Int. 2010 Oct; 78(7):698-704.

32 Kopp JB, Nelson GW, Sampath K, Johnson RC, Genovese G, An P, et al. APOL1 genetic variants in focal segmental glomerulosclerosis and HIV-associated nephropathy. J Am Soc Nephrol. 2011 Nov;22(11):2129-37.

33 Kopp JB, Winkler CA, Zhao X, Radeva MK Gassman JJ, D'Agati VD, et al. Clinical features and histology of apolipoprotein L1-associated nephropathy in the FSGS clinical trial. J Am Soc Nephrol. 2015 Jun;26(6):1443-8.

34 Freedman BI, Langefeld CD. The new era of APOL1-associated glomerulosclerosis. Nephrol Dial Transplant. 2012 Apr;27(4): 1288-91.

35 Snoek R, Nguyen TQ, van der Zwaag B, van Zuilen AD, Kruis HME, van Gils-Verrij LA, et al. Importance of genetic diagnostics in adult-onset focal segmental glomerulosclerosis. Nephron. 2019;142(4):351-8.

36 Landini S, Mazzinghi B, Becherucci F, Allinovi M, Provenzano A, Palazzo V, et al. Reverse phenotyping after whole-exome sequencing in steroid-resistant nephrotic syndrome. Clin J Am Soc Nephrol. 2020 Jan 7; 15(1):89-100.

37 Stokes MB, D'Agati VD. Morphologic variants of focal segmental glomerulosclerosis and their significance. Adv Chronic Kidney Dis. 2014 Sep;21(5):400-7.

38 Schachter AD. Computational simulation of renal biopsy accuracy in focal segmental glomerulosclerosis. Pediatr Nephrol. 2006 Jul; 21(7):953-7.

39 Francis A, Trnka P, McTaggart SJ. Long-term outcome of kidney transplantation in recipients with focal segmental glomerulosclerosis. Clin J Am Soc Nephrol. 2016 Nov 7;11(11): 2041-6.
Update in Focal Segmental

Glomerulosclerosis
Nephron 2020;144:413-427

DOI: $10.1159 / 000508099$ 
40 Ponticelli C. Recurrence of focal segmental glomerular sclerosis (FSGS) after renal transplantation. Nephrol Dial Transplant. 2010 Jan;25(1):25-31.

41 Maas RJ, Deegens JK, van den Brand JA, Cornelissen EA, Wetzels JF. A retrospective study of focal segmental glomerulosclerosis: clinical criteria can identify patients at high risk for recurrent disease after first renal transplantation. BMC Nephrol. 2013 Feb 22;14:47.

42 Ding WY, Koziell A, McCarthy HJ, Bierzynska A, Bhagavatula MK, Dudley JA, et al. Initial steroid sensitivity in children with steroid-resistant nephrotic syndrome predicts post-transplant recurrence. J Am Soc Nephrol. 2014 Jun;25(6):1342-8.

43 Campise M, Favi E, Messa P. Clinical outcomes of prophylactic and therapeutic plasmapheresis in adult deceased-donor kidney transplant recipients with primary focal segmental glomerulosclerosis. Exp Clin Transplant. 2019 Aug;17(4):461-9. Available from: http://www.ectrx.org/detail/archive/2019/ 17/4/0/461/0.http://dx.doi.org/10.6002/ ect.2018.0106

44 Auñón P, Polanco N, Pérez-Sáez MJ, Rodrigo E, Sancho A, Pascual J, et al. Pre-emptive rituximab in focal and segmental glomerulosclerosis patients at risk of recurrence after kidney transplantation. Clin Kidney J. 2019 Oct 3;sfz120.

45 Chailimpamontree W, Dmitrienko S, Li G, Balshaw R, Magil A, Shapiro RJ, et al. Probability, predictors, and prognosis of posttransplantation glomerulonephritis. J Am Soc Nephrol. 2009 Apr;20(4):843-51.

46 Ponticelli C, Moroni G, Glassock RJ. De Novo glomerular diseases after renal transplantation. Clin J Am Soc Nephrol. 2014 Aug 7; 9(8):1479-87.

47 Letavernier E, Bruneval P, Vandermeersch S, Perez J, Mandet C, Belair MF, et al. Sirolimus interacts with pathways essential for podocyte integrity. Nephrol Dial Transplant. 2009 Feb; 24(2):630-8.

48 Cosio FG, Frankel WL, Pelletier RP, Pesavento TE, Henry ML, Ferguson RM. Focal segmental glomerulosclerosis in renal allografts with chronic nephropathy: implications for graft survival. Am J Kidney Dis. 1999 Oct; 34(4):731-8.

49 Shen WW, Chen HM, Chen H, Xu F, Li LS, Liu ZH. Obesity-related glomerulopathy: body mass index and proteinuria. Clin J Am Soc Nephrol. 2010 Aug;5(8):1401-9.

50 Korbet SM. Treatment of primary FSGS in adults. J Am Soc Nephrol. 2012 Nov;23(11): 1769-76.

51 Fornoni A, Sageshima J, Wei C, MerscherGomez S, Aguillon-Prada R, Jauregui AN, et al. Rituximab targets podocytes in recurrent focal segmental glomerulosclerosis. Sci Transl Med. 2011 Jun 1;3(85):85ra46.
52 Faul C, Donnelly M, Merscher-Gomez S, Chang YH, Franz S, Delfgaauw J, et al. The actin cytoskeleton of kidney podocytes is a direct target of the antiproteinuric effect of cyclosporine A. Nat Med. 2008 Sep;14(9):931-8.

53 Glassock RJ. Prophylactic anticoagulation in nephrotic syndrome: a clinical conundrum. J Am Soc Nephrol. 2007 Aug;18(8):2221-5.

54 Fernandez-Juarez G, Villacorta J, Ruiz-Roso G, Panizo N, Martinez-Marín I, Marco H, et al. Therapeutic variability in adult minimal change disease and focal segmental glomerulosclerosis. Clin Kidney J. 2016 Jun;9(3):3816.

55 Cattran DC, Rao P. Long-term outcome in children and adults with classic focal segmental glomerulosclerosis. Am J Kidney Dis. 1998 Jul;32(1):72-9.

56 Stirling CM, Mathieson P, Boulton-Jones JM, Feehally J, Jayne D, Murray HM, et al. Treatment and outcome of adult patients with primary focal segmental glomerulosclerosis in five UK renal units. QJM. 2005 Jun;98(6):443-9.

57 Ponticelli C, Villa M, Banfi G, Cesana B, Pozzi C, Pani A, et al. Can prolonged treatment improve the prognosis in adults with focal segmental glomerulosclerosis? Am J Kidney Dis. 1999 Oct;34(4):618-25.

58 Nagai R, Cattran DC, Pei Y. Steroid therapy and prognosis of focal segmental glomerulosclerosis in the elderly. Clin Nephrol. 1994 Jul; 42(1):18-21.

59 Goumenos DS, Tsagalis G, El Nahas AM, Shortland JR, Davlouros P, Vlachojannis JG, et al. Immunosuppressive treatment of idiopathic focal segmental glomerulosclerosis: a five-year follow-up study. Nephron Clin Pract. 2006;104(2):c75-82.

60 Laurin LP, Gasim AM, Poulton CJ, Hogan SL, Jennette JC, Falk RJ, et al. Treatment with glucocorticoids or calcineurin inhibitors in primary FSGS. Clin J Am Soc Nephrol. 2016 Mar 7;11(3):386-94.

61 Senthil Nayagam L, Ganguli A, Rathi M, Kohli HS, Gupta KL, Joshi K, et al. Mycophenolate mofetil or standard therapy for membranous nephropathy and focal segmental glomerulosclerosis: a pilot study. Nephrol Dial Transplant. 2008 Jun;23(6):1926-30.

62 Troyanov S, Wall CA, Miller JA, Scholey JW, Cattran DC. Focal and segmental glomerulosclerosis: definition and relevance of a partial remission. J Am Soc Nephrol. 2005 Apr; 16(4):1061-8.

63 Ponticelli C, Rizzoni G, Edefonti A, Altieri P, Rivolta E, Rinaldi S, et al. A randomized trial of cyclosporine in steroid-resistant idiopathic nephrotic syndrome. Kidney Int. 1993 Jun; 43(6):1377-84.

64 Cattran DC, Appel GB, Hebert LA, Hunsicker LG, Pohl MA, Hoy WE, et al. A randomized trial of cyclosporine in patients with steroidresistant focal segmental glomerulosclerosis. North America nephrotic syndrome study group. Kidney Int. 1999 Dec;56(6):2220-6.
65 Hodson EM, Wong SC, Willis NS, Craig JC. Interventions for idiopathic steroid-resistant nephrotic syndrome in children. Cochrane Database Syst Rev. 2019 Nov 21;10(11): CD003594.

66 Gipson DS, Trachtman H, Kaskel FJ, Greene TH, Radeva MK, Gassman JJ, et al. Clinical trial of focal segmental glomerulosclerosis in children and young adults. Kidney Int. 2011 Oct;80(8):868-78.

67 Cattran DC, Wang MM, Appel G, Matalon A, Briggs W. Mycophenolate mofetil in the treatment of focal segmental glomerulosclerosis. Clin Nephrol. 2004 Dec;62(6):405-11.

68 Segarra A, Amoedo ML, Martinez Garcia JM, Pons S, Praga M, Garcia EI, et al. Efficacy and safety of "rescue therapy" with mycophenolate mofetil in resistant primary glomerulonephritis: a multicenter study. Nephrol Dial Transplant. 2007 May;22(5):1351-60.

69 Fernandez-Fresnedo G, Segarra A, González E, Alexandru S, Delgado R, Ramos N, et al. Rituximab treatment of adult patients with steroid-resistant focal segmental glomerulosclerosis. Clin J Am Soc Nephrol. 2009 Aug; 4(8):1317-23.

70 Kamei K, Ishikura K, Sako M, Ito S, Nozu K, Iijima K. Rituximab therapy for refractory steroid-resistant nephrotic syndrome in children. Pediatr Nephrol. 2020 Jan;35(1):17-24.

71 Heering P, Braun N, Müllejans R, Ivens K, Zäuner I, Fünfstück R, et al. Cyclosporine A and chlorambucil in the treatment of idiopathic focal segmental glomerulosclerosis. Am J Kidney Dis. 2004 Jan;43(1):10-8.

72 Sadowski CE, Lovric S, Ashraf S, Pabst WL, Gee HY, Kohl S, et al. A single-gene cause in $29.5 \%$ of cases of steroid-resistant nephrotic syndrome. J Am Soc Nephrol. 2015 Jun;26(6): 1279-89.

73 Ponticelli C, Edefonti A, Ghio L, Rizzoni G, Rinaldi S, Gusmano R, et al. Cyclosporin versus cyclophosphamide for patients with steroid-dependent and frequently relapsing idiopathic nephrotic syndrome: a multicentre randomized controlled trial. Nephrol Dial Transplant. 1993;8(12):1326-32.

74 Kronbichler A, Kerschbaum J, FernandezFresnedo G, Hoxha E, Kurschat CE, Busch M, et al. Rituximab treatment for relapsing minimal change disease and focal segmental glomerulosclerosis: a systematic review. Am J Nephrol. 2019 Oct 3;39(4):322-30.

75 Ochi A, Takei T, Nakayama K, Iwasaki C, Kamei D, Tsuruta Y, et al. Rituximab treatment for adult patients with focal segmental glomerulosclerosis. Intern Med. 2012;51(7):75962.

76 Ruggenenti P, Ruggiero B, Cravedi P, Vivarelli M, Massella L, Marasà M, et al. Rituximab in steroid-dependent or frequently relapsing idiopathic nephrotic syndrome. J Am Soc Nephrol. 2014 Apr;25(4):850-63. 
77 Basu B. Ofatumumab for rituximab-resistant nephrotic syndrome. N Engl J Med. 2014 Mar 27;370(13):1268-70.

78 Hogan J, Bomback AS, Mehta K, Canetta PA, Rao MK, Appel GB, et al. Treatment of idiopathic FSGS with adrenocorticotropic hormone gel. Clin J Am Soc Nephrol. 2013 Dec; 8(12):2072-81.

79 Yu CC, Fornoni A, Weins A, Hakroush S, Maiguel D, Sageshima J, et al. Abatacept in B7-1-positive proteinuric kidney disease. N Engl J Med. 2013 Dec 19;369(25):2416-23.
80 Delville M, Baye E, Durrbach A, Audard V, Kofman T, Braun L, et al. B7-1 Blockade does not improve post-transplant nephrotic syndrome caused by recurrent FSGS. J Am Soc Nephrol. 2016 Aug;27(8):2520-7.

81 Garin EH, Reiser J, Cara-Fuentes G, Wei C, Matar D, Wang H, et al. Case series: CTLA4IgG1 therapy in minimal change disease and focal segmental glomerulosclerosis. Pediatr Nephrol. 2015 Mar;30(3):469-77.

82 Trachtman H, Nelson P, Adler S, Campbell $\mathrm{KN}$, Chaudhuri A, Derebail VK, et al. DUET: a phase 2 study evaluating the efficacy and safety of sparsentan in patients with FSGS. J Am Soc Nephrol. 2018;29(11): 2745-54.
83 Mitwalli AH. Adding plasmapheresis to corticosteroids and alkylating agents: does it benefit patients with focal segmental glomerulosclerosis? Nephrol Dial Transplant. 1998 Jun; 13(6):1524-8.

84 Feld SM, Figueroa P, Savin V, Nast CC, Sharma R, Sharma M, et al. Plasmapheresis in the treatment of steroid-resistant focal segmental glomerulosclerosis in native kidneys. Am J Kidney Dis. 1998 Aug;32(2):230-7.

85 Muso E, Mune M, Yorioka N, Nishizawa Y, Hirano T, Hattori M, et al. Beneficial effect of low-density lipoprotein apheresis (LDL-A) on refractory nephrotic syndrome (NS) due to focal glomerulosclerosis (FGS). Clin Nephrol. 2007 Jun;67(6):341-4. 